\title{
Hausdorff measures and two point set extensions
}

\author{
by \\ Jan J. Dij kstra (Tuscaloosa, Ala.), \\ Kenneth Kunen (Madison, Wisc.) and Jan van Mill (Amsterdam)
}

\begin{abstract}
We investigate the following question: under which conditions is a $\sigma$ compact partial two point set contained in a two point set? We show that no reasonable measure or capacity (when applied to the set itself) can provide a sufficient condition for a compact partial two point set to be extendable to a two point set. On the other hand, we prove that under Martin's Axiom any $\sigma$-compact partial two point set such that its square has Hausdorff 1-measure zero is extendable.
\end{abstract}

1. Introduction. A planar set is called a two point set if every line intersects the set in exactly two points, and a partial two point set if every line intersects the set in at most two points. We call a partial two point set extendable if it is a subset of some two point set. The existence of two point sets is due to Mazurkiewicz [7]. His proof shows that every partial two point set with cardinality less than $\mathfrak{c}$ is extendable. A circle is the standard example of a nonextendable partial two point set.

In [6, 9, Problem 1070] R. D. Mauldin asked whether every compact zero-dimensional partial two point set can be extended to a two point set. It was shown by two of the authors in [2] and independently by Mauldin in [7] that there exist partial two point Cantor sets that are not extendable to two point sets. In both papers the proof rests on the fact that the examples have positive linear Lebesgue measure. It is therefore natural to ask whether there exist nonextendable partial two point compacta with vanishing linear measure (or Hausdorff 1-measure zero). In addition, what about Hausdorff dimension zero or logarithmic capacity zero? In this paper the authors answer these questions by showing that no reasonable measure or capacity (when applied to the set itself) can provide a sufficient condition for a compact partial two point set to be extendable to a two point set.

1991 Mathematics Subject Classification: 28A78, 54G99.

The second author supported in part by NSF grant DMS-9704520. 
These results can be found in Section 2. In Section 3 we present sufficient conditions for a partial two point set to be extendable. For example, under Martin's Axiom any $\sigma$-compact partial two point set such that its square has Hausdorff 1-measure zero is extendable.

2. Nonextendable sets. We denote the space of nonempty compacta in a metric space $X$ equipped with the usual Hausdorff metric by $\mathcal{K}(X)$. The space of projection directions in the plane is the circle $\Theta=\mathbb{R} / \pi \mathbb{Z}$. If $\theta \in \Theta$ then $p_{\theta}$ is the projection of the plane onto the line through the origin that is perpendicular to $\theta$. Obviously, the function $p: \Theta \times \mathbb{R}^{2} \rightarrow \mathbb{R}^{2}$ defined by $p(\theta, u)=p_{\theta}(u)$ is a continuous mapping and hence it generates a continuous map from $\Theta \times \mathcal{K}\left(\mathbb{R}^{2}\right)$ to $\mathcal{K}\left(\mathbb{R}^{2}\right)$. If $u$ is a nonzero vector in the plane then $\varphi(u) \in \Theta$ stands for the direction parallel to $u$. If $u$ and $v$ are two distinct points in the plane then $L(u, v)$ stands for the line through $u$ and $v$. If $A$ is a planar set then

$$
\mathfrak{L}(A)=\bigcup\{L(u, v): u, v \in A \text { and } u \neq v\}
$$

and

$$
\mathfrak{B}(A)=\{p(\varphi(u-v), u): u, v \in A \text { and } u \neq v\} .
$$

Observe that $p(\varphi(u-v), u)$ is the point of intersection of $L(u, v)$ with the line through 0 that is perpendicular to $L(u, v)$ and that in terms of the dot product in $\mathbb{R}^{2}$ we have

$$
p(\varphi(u-v), u)=\frac{((v-u) \cdot v) u+((u-v) \cdot u) v}{|u-v|^{2}} .
$$

If $v \in \mathbb{R}^{2} \backslash\{0\}$ then $C_{v}$ stands for the circle that has the line segment from 0 to $v$ as diameter.

Lemma 2.1. If $A \subset \mathbb{R}^{2}$ then

$$
\left\{v \in \mathbb{R}^{2} \backslash\{0\}: C_{v} \cap \mathfrak{B}(A) \backslash\{0\} \neq \emptyset\right\} \subset \mathfrak{L}(A) .
$$

Pro of. Let $u \in C_{v} \cap \mathfrak{B}(A)$ with $u \neq 0$. Then there exists a line $\ell$ through $u$ that is perpendicular to the line through 0 and $u$ and that intersects $A$ in two distinct points $a$ and $b$ (see Figure 1).

Since the angle at $u$ is $90^{\circ}$ we see by elementary planar geometry that $\ell=L(a, b)$ intersects $C_{v}$ at $v$.

Let $S^{1}$ be the unit circle centered at the origin. If $A \subset S^{1}$ then we simply have $\mathfrak{B}(A)=\left\{\frac{1}{2}(u+v): u, v \in A\right.$ and $\left.u \neq v\right\}$.

We will show in the next section (see Proposition 3.7 ) that the nonextendable elements of $\mathcal{K}\left(S^{1}\right)$ form a category I subset of $\mathcal{K}\left(S^{1}\right)$. So finding nonextendable elements in $\mathcal{K}\left(S^{1}\right)$ is relatively hard because the set to choose from is small and standard Baire category arguments will not work. However, our next result gives us a rich supply of nonextendable compact subsets 


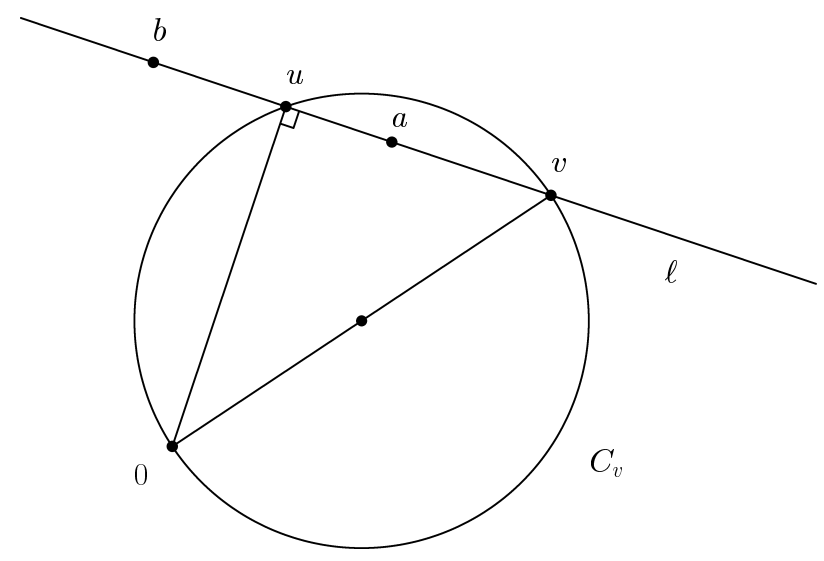

Figure 1

of $S^{1}$. Since the concept of (partial) two point set is invariant under affine transformations the results we obtain for $S^{1}$ apply to any ellipse.

TheOREM 2.2. Every dense $G_{\delta}$-subset of $\mathcal{K}\left(S^{1}\right)$ contains two elements with a nonextendable union.

Pro of. Let $G_{0} \supset G_{1} \supset \ldots$ be a sequence of dense open subsets of $\mathcal{K}\left(S^{1}\right)$ and define $G=\bigcap_{n=0}^{\infty} G_{n}$. Let $D^{1}=\left\{u \in \mathbb{R}^{2}:|u| \leq 1\right\}$ and $D_{0}^{1}=D^{1} \backslash\{0\}$. Define the continuous maps $\alpha, \beta: D_{0}^{1} \rightarrow S^{1}$ as in Figure 2 .

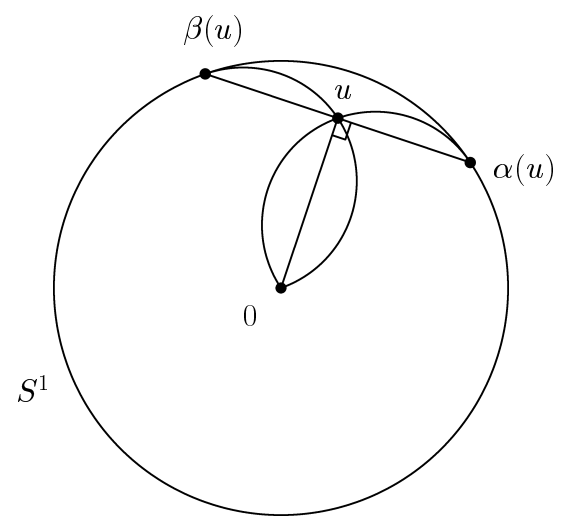

Figure 2

To be precise $\alpha$ and $\beta$ are given by

$$
\alpha(u)=(x+t y, y-t x) \text { and } \beta(u)=(x-t y, y+t x),
$$

where $u=(x, y) \in D_{0}^{1}$ and $t=\sqrt{1-|u|^{2}} /|u|$. Then for each $u \in D_{0}^{1}$ we 
have

$$
p(\varphi(\alpha(u)-\beta(u)), \alpha(u))=\frac{1}{2}(\alpha(u)+\beta(u))=u .
$$

The semicircles with diameter 1 in Figure 2 correspond to preimages of the form $\alpha^{-1}(w)$ and $\beta^{-1}\left(w^{\prime}\right)$ where $w, w^{\prime} \in S^{1}$. The building blocks of our construction will be segments of such semicircles.

We shall construct an element $A$ of $\mathcal{K}\left(S^{1}\right)$ such that every circle through the origin with diameter at least 2 intersects $\mathfrak{B}(A)$ in a point that is not the origin. Then by Lemma 2.1 we have $\left\{v \in \mathbb{R}^{2}:|v| \geq 2\right\} \subset \mathfrak{L}(A)$. So if we add any point $v$ with $|v| \geq 2$ to $A$ then the resulting set is no longer a partial two point set and hence $A$ is not extendable to a two point set.

Let $d$ be the arc length metric on $S^{1}$. Note that $u \mapsto(\alpha(u), \beta(u))$ is an imbedding of $D_{0}^{1}$ in the product $S^{1} \times S^{1}$. This observation allows us to define a metric $\varrho$ on $D_{0}^{1}$ by pulling back the max metric of $S^{1} \times S^{1}$ : for $u, v \in D_{0}^{1}$,

$$
\varrho(u, v)=\max \{d(\alpha(u), \alpha(v)), d(\beta(u), \beta(v))\} .
$$

Let $\widetilde{d}$ and $\widetilde{\varrho}$ be the corresponding Hausdorff metrics on $\mathcal{K}\left(S^{1}\right)$ and $\mathcal{K}\left(D_{0}^{1}\right)$ respectively. If $X$ is a subset of $S^{1}$ and $\varepsilon>0$, let $U_{\varepsilon}(X)=\left\{w \in S^{1}\right.$ : $d(w, X) \leq \varepsilon\}$. If $w \in S^{1}$ then $U_{\varepsilon}(w)=U_{\varepsilon}(\{w\})$. If $u, v \in S^{1}$ then $[u, v]$ stands for the closed segment of $S^{1}$ from $u$ to $v$ (with counterclockwise orientation). If $X \in \mathcal{K}\left(S^{1}\right)$ then $\widetilde{U}_{\varepsilon}(X) \subset \mathcal{K}\left(S^{1}\right)$ denotes the closed $\varepsilon$-ball around $X$ with respect to the Hausdorff metric $\widetilde{d}$.

Let $\mathcal{C}$ be the collection of all semicircles $C$ with diameter at least 2 such that one of the endpoints is the origin. We shall construct inductively a sequence $B_{0}, B_{1}, \ldots$ in $\mathcal{K}\left(D_{0}^{1}\right)$ and a sequence $\varepsilon_{0}, \varepsilon_{1}, \ldots$ of positive numbers such that

(1) $\varepsilon_{n} \leq \varepsilon_{n-1} / 2$

(2) $\widetilde{\varrho}\left(B_{n}, B_{n-1}\right) \leq \varepsilon_{n-1} / 2$,

(3) $B_{n}$ intersects every element of $\mathcal{C}$,

(4) $\widetilde{U}_{\varepsilon_{n}}\left(\alpha\left(B_{n}\right)\right) \subset G_{n}$ if $n$ is even,

(5) $\widetilde{U}_{\varepsilon_{n}}\left(\beta\left(B_{n}\right)\right) \subset G_{n}$ if $n$ is odd,

(6) if $n$ is even then $B_{n}$ is a finite union of sets of the form $\alpha^{-1}(p) \cap$ $\beta^{-1}([u, v])$, where $p, u, v \in S^{1}$,

(7) if $n$ is odd then $B_{n}$ is a finite union of sets of the form $\beta^{-1}(p) \cap$ $\alpha^{-1}([u, v])$, where $p, u, v \in S^{1}$.

Let $F$ be a $\delta$-net in $S^{1}$, i.e. a finite subset of $S^{1}$ with $\widetilde{d}\left(F, S^{1}\right) \leq \delta$. Consider the set

$$
P_{F}=\alpha^{-1}(F) \cap\left\{u \in D^{1}: \frac{1}{4} \leq|u| \leq \frac{3}{4}\right\}
$$

(see Figure 3). 


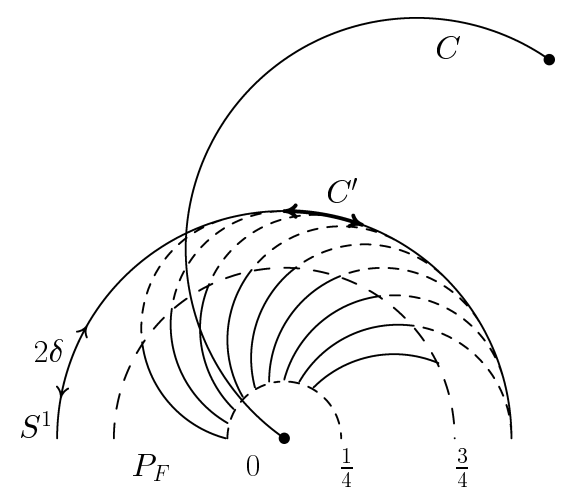

Figure 3

We choose $\delta$ so small that for any $\delta$-net $F$ and any semicircle $C \in \mathcal{C}, P_{F}$ and $C$ intersect. This is possible since there exists a $\delta$ such that for every $C \in \mathcal{C}$, the projection $C^{\prime}=\alpha\left(\left\{u \in C: \frac{1}{4} \leq|u| \leq \frac{3}{4}\right\}\right)$ is an interval of length at least $2 \delta$. Since the finite sets are dense in $\mathcal{K}\left(S^{1}\right)$ we may select a $\delta$-net $F \in G_{0}$. We put $B_{0}=P_{F}$ and we select an $\varepsilon_{0}>0$ such that $\widetilde{U}_{\varepsilon_{0}}(F)$ is contained in $G_{0}$ and such that for any $X \in \mathcal{K}\left(D_{0}^{1}\right), \widetilde{\varrho}\left(X, B_{0}\right) \leq \varepsilon_{0}$ implies that $X$ is contained in $D^{\prime}=\left\{w \in D^{1}: \frac{1}{8} \leq|w| \leq \frac{7}{8}\right\}$. Consequently, every $B_{n}$ will be a subset of $D^{\prime}$. The applicable induction hypotheses are obviously satisfied.

Let us assume that $B_{n}$ and $\varepsilon_{n}$ have been found. We now describe the procedure for constructing $B_{n+1}$, assuming that $n$ is even. The procedure for $n$ odd is simply the mirror image (interchange $\alpha$ and $\beta$ ). The set $B_{n}$ is the union of the finite collection $\left\{E_{1}, \ldots, E_{k}\right\}$, where $E_{j}=\alpha^{-1}\left(p_{j}\right) \cap$ $\beta^{-1}\left(\left[u_{j}, v_{j}\right]\right)$ for some $p_{j}, u_{j}, v_{j} \in S^{1}$. Consider $E_{j}$ and pick a $\delta \leq \varepsilon_{n} / 4$. We select a $\gamma_{j} \leq \delta$ such that every finite $F \subset S^{1}$ with $\widetilde{d}\left(F, U_{\delta}\left(\left[u_{j}, v_{j}\right]\right)\right) \leq \gamma_{j}$ has the property that

$$
Q_{j}(F)=\alpha^{-1}\left(U_{\delta}\left(p_{j}\right)\right) \cap \beta^{-1}(F)
$$

intersects every $C \in \mathcal{C}$ that intersects $E_{j}$ (see Figure 4 ).

Let $\gamma=\min \left\{\gamma_{1}, \ldots, \gamma_{k}\right\}$. Select a finite set $F \in G_{n+1}$ such that

$$
\widetilde{d}\left(F, \bigcup_{j=1}^{k} U_{\delta}\left(\left[u_{j}, v_{j}\right]\right)\right)<\gamma .
$$

We define the compactum

$$
B_{n+1}=\bigcup_{j=1}^{k} Q_{j}\left(F_{j}\right),
$$

where $F_{j}=F \cap U_{\delta+\gamma}\left(\left[u_{j}, v_{j}\right]\right)$. Let $\varepsilon_{n+1}>0$ be such that $\varepsilon_{n+1}<\varepsilon_{n} / 2$ and $\widetilde{U}_{\varepsilon_{n+1}}(F) \subset G_{n+1}$. 


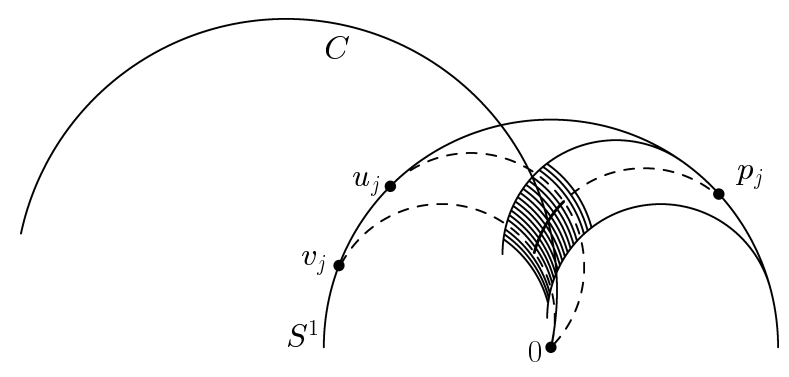

Figure 4

Since every $Q_{j}\left(F_{j}\right)$ is obviously a finite union of sets of the form $\beta^{-1}(w) \cap$ $\alpha^{-1}\left(U_{\delta}(p)\right)$ the set $B_{n+1}$ satisfies condition (7). Condition (1) is trivially true. Note that $\beta\left(Q_{j}\left(F_{j}\right)\right)=F_{j}$ and that $F=\bigcup_{j=1}^{k} F_{j}$. Consequently, $\beta\left(B_{n+1}\right)=F$ and condition (5) is satisfied. In addition, $\widetilde{d}\left(F_{j}, U_{\delta}\left(\left[u_{j}, v_{j}\right]\right)\right) \leq$ $\gamma \leq \gamma_{j}$ so if $C \in \mathcal{C}$ intersects $E_{j}$ then $C$ intersects $Q_{j}\left(F_{j}\right)$ and $B_{n+1}$. By hypothesis, every $C \in \mathcal{C}$ intersects some $E_{j}$ and hence (3) is valid for $n+1$. We have

$$
\widetilde{d}\left(\left\{p_{j}\right\}, U_{\delta}\left(p_{j}\right)\right)=\delta \quad \text { and } \quad \tilde{d}\left(\left[u_{j}, v_{j}\right], F_{j}\right) \leq \delta+\gamma \leq 2 \delta .
$$

Since $E_{j}$ and $Q_{j}\left(F_{j}\right)$ are essentially Cartesian products of these sets and $\widetilde{\varrho}$ corresponds to the product max metric it follows that $\widetilde{\varrho}\left(E_{j}, Q_{j}\left(F_{j}\right)\right) \leq$ $2 \delta \leq \varepsilon_{n} / 2$ (see Figure 4 ). Consequently, we have $\widetilde{\varrho}\left(B_{n}, B_{n+1}\right) \leq \varepsilon_{n} / 2$ and the induction is complete.

Induction hypotheses (1) and (2) show that $B_{0}, B_{1}, \ldots$ is a Cauchy sequence with respect to $\widetilde{\varrho}$. In addition they show that $\widetilde{\varrho}\left(B_{0}, B_{n}\right) \leq \varepsilon_{0}$ for each $n$ and hence we have a Cauchy sequence in the compact space $\mathcal{K}\left(D^{\prime}\right)$. Put

$$
B=\lim _{n \rightarrow \infty} B_{n} \in \mathcal{K}\left(D^{\prime}\right) \quad \text { and } \quad A=\alpha(B) \cup \beta(B) \in \mathcal{K}\left(S^{1}\right) .
$$

Obviously, we have $B \subset \mathfrak{B}(A)$. Since every $C \in \mathcal{C}$ intersects every $B_{n}$ we deduce by compactness that $B$ intersects every $C \in \mathcal{C}$. So $\mathfrak{B}(A)$ intersects every element of $\mathcal{C}$ in a point other than the origin. As argued above, this result implies that $A$ is not extendable.

Let $n$ be even and $k \in \mathbb{N}$. Observe that

$$
\widetilde{d}\left(\alpha\left(B_{n}\right), \alpha\left(B_{n+k}\right)\right) \leq \widetilde{\varrho}\left(B_{n}, B_{n+k}\right) \leq \sum_{i=1}^{k} \varepsilon_{n} 2^{-i} \leq \varepsilon_{n} .
$$

So $\alpha\left(B_{n+k}\right) \in \widetilde{U}_{\varepsilon_{n}}\left(\alpha\left(B_{n}\right)\right)$ and $\alpha(B)=\lim _{k \rightarrow \infty} \alpha\left(B_{n+k}\right) \in \widetilde{U}_{\varepsilon_{n}}\left(B_{n}\right)$. By condition (4) we have $\alpha(B) \in G_{n}$ and hence $\alpha(B) \in \bigcap_{n=0}^{\infty} G_{2 n}=G$. The same argument for $n$ odd yields $\beta(B) \in G$. 
Theorem 2.2 is sharp in the sense that there exist dense $G_{\delta}$-subsets of $\mathcal{K}\left(S^{1}\right)$ consisting entirely of extendable elements (see Proposition 3.7).

A real-valued function is called upper semicontinuous if the preimage of every interval of the form $(-\infty, t)$ is open. We call a function $\gamma: \mathcal{K}(X) \rightarrow$ $[0, \infty]$ null-subadditive if $\gamma(A)=\gamma(B)=0$ implies $\gamma(A \cup B)=0$. Measures and capacities satisfy these conditions.

Theorem 2.3. If $\gamma: \mathcal{K}\left(S^{1}\right) \rightarrow[0, \infty]$ is a null-subadditive and upper semicontinuous function that vanishes on finite sets then there exists a nonextendable $C \in \mathcal{K}\left(S^{1}\right)$ with $\gamma(C)=0$.

Proof. The set $\gamma^{-1}(0)$ is a dense $G_{\delta}$ in $\mathcal{K}\left(S^{1}\right)$ that is closed under unions.

Note that instead of upper semicontinuity it suffices to know that sets of the form $\gamma^{-1}([0, \varepsilon))$ are neighbourhoods of sets with $\gamma$ equal to zero.

COROLlary 2.4. There exists a nonextendable element of $\mathcal{K}\left(S^{1}\right)$ with linear Lebesgue measure zero.

It is well known that capacities satisfy the premise of Theorem 3.2. The most interesting capacity in the plane is the logarithmic or Newtonian capacity (see e.g. $[4,12]$ ).

COROLlary 2.5. There exists a nonextendable element of $\mathcal{K}\left(S^{1}\right)$ with logarithmic capacity zero.

Let us have a look at Hausdorff type measures. For every $h:\left[0, t_{0}\right] \rightarrow$ $[0, \infty)$ we define the $h$-measure $m_{h}$ of a subset $X$ of $\mathbb{R}^{n}$ by

$$
\begin{aligned}
& m_{h}(X)=\lim _{\varepsilon \searrow 0} \inf \left\{\sum_{i=1}^{\infty} h\left(\operatorname{diam}\left(A_{i}\right)\right):\left\{A_{i}: i \in \mathbb{N}\right\}\right. \text { is a covering } \\
&\text { in } \left.\mathbb{R}^{n} \text { of } X \text { with sets of diameter at most } \varepsilon\right\} .
\end{aligned}
$$

$\mathbb{R}^{n}$ is assumed to be equipped with the metric that is generated by the standard norm $\sqrt{\sum_{i=1}^{n} x_{i}^{2}}$. If $s>0$ then the Hausdorff $s$-measure $\mathcal{H}^{s}$ equals $m_{h}$, where $h(t)=t^{s}$. Recall that the Hausdorff dimension of $X$ is defined by

$$
\operatorname{dim}_{\mathrm{H}}(X)=\inf \left\{s: \mathcal{H}^{s}(X)=0\right\} .
$$

Since a set with vanishing logarithmic capacity has Hausdorff dimension zero (see [4, Theorem 3.13]), we have

COROLlary 2.6. There exists a nonextendable element of $\mathcal{K}\left(S^{1}\right)$ with Hausdorff dimension zero.

Note that if $A \in \mathcal{K}\left(S^{1}\right)$ has $\operatorname{dim}_{\mathrm{H}}(A)=0$ then $\operatorname{dim}_{\mathrm{H}}\left(A \times S^{1}\right)=1$ and hence $\operatorname{dim}_{\mathrm{H}}(A \times A) \leq 1$. So we have the following result whose significance will become apparent in the next section. 
Corollary 2.7. There exists a nonextendable $A \in \mathcal{K}\left(S^{1}\right)$ such that $\operatorname{dim}_{\mathrm{H}}(A \times A) \leq 1$

Corollary 2.6 also follows if we substitute $h(t)=1 / \log (1 / t)$ into the following general statement about $h$-measures.

COROLlary 2.8. There exists a nonextendable $C \in \mathcal{K}\left(S^{1}\right)$ with $m_{h}(C)=0$ if and only if $\liminf _{t \searrow 0} h(t)=0$.

P r o of. If $\liminf \operatorname{ino}_{t} h(t)>0$ and $m_{h}(C)=0$ then $C$ is countable. Every partial two point set with cardinality $<\mathfrak{c}$ is extendable.

Assume that $\liminf \operatorname{in}_{t} h(t)=0$. Let for each $n \in \mathbb{N}$ the set $O_{n}$ be the interior in $\mathcal{K}\left(S^{1}\right)$ of the collection

$$
\begin{aligned}
\left\{C \in \mathcal{K}\left(S^{1}\right):\right. & \text { there is a covering }\left\{A_{i}: i \in \mathbb{N}\right\} \text { in } \mathbb{R}^{2} \text { of } C \\
& \text { with sets of diameter at most } t_{0} / n \\
& \text { such that } \left.\sum_{i=1}^{\infty} h\left(\operatorname{diam}\left(A_{i}\right)\right) \leq 1 / n\right\} .
\end{aligned}
$$

Obviously, every element of $G=\bigcap_{n=1}^{\infty} O_{n}$ has $m_{h}$ equal to 0 . Let $F \in \mathcal{K}\left(S^{1}\right)$ be a finite set with $m$ points and let $n \in \mathbb{N}$. Select an $\varepsilon>0$ such that $\varepsilon \leq t_{0} / n$ and $h(\varepsilon) \leq(m n)^{-1}$. Let $C \in \mathcal{K}\left(S^{1}\right)$ be a set whose distance from $F$ in the Hausdorff metric is less than $\varepsilon / 2$. Then $C$ can be covered by intervals $A_{1}, \ldots, A_{m}$ each with diameter equal to $\varepsilon$. Consequently,

$$
\sum_{i=1}^{m} h\left(\operatorname{diam}\left(A_{i}\right)\right)=m h(\varepsilon) \leq \frac{1}{n}
$$

and we may conclude that $F \in O_{n}$. So $G$ is a $G_{\delta}$ in $\mathcal{K}\left(S^{1}\right)$ which contains all finite sets and Theorem 2.2 applies. It is easily seen that if $A, B \in O_{2 n}$ then $A \cup B \in O_{n}$. This implies that $G$ is closed under unions.

Corollary 2.8 allows us to improve upon Corollary 2.7 which follows if we substitute $h(t)=t / \log (1 / t)$ in

Proposition 2.9. If every $A \in \mathcal{K}\left(S^{1}\right)$ with $m_{h}(A \times A)=0$ is extendable then the following two equivalent statements are valid:

(1) $m_{h}(X)=0$ implies $\mathcal{H}^{1}(X)=0$ for every $X$,

(2) $\liminf \operatorname{i\searrow 0}_{t} h(t) / t>0$.

Proof. First we verify the equivalence of (1) and (2). Assume first that (2) is valid. Then there are $\varepsilon, \delta>0$ such that $h(t) \geq \delta t$ for $0<$ $t<\varepsilon$. Consequently, $m_{h}(X) \geq \delta \mathcal{H}^{1}(X)$ for any $X$, which implies statement (1). Consider now the case $\liminf _{t \backslash 0} h(t) / t=0$. Select a sequence $t_{1}, t_{2}, \ldots$ of positive numbers less than 1 and $t_{0}$ such that $\lim _{i \rightarrow \infty} t_{i}=0$ and $\lim _{i \rightarrow \infty} h\left(t_{i}\right) / t_{i}=0$. If $I$ is the unit interval $[0,1]$ then $\mathcal{H}^{1}(I)=1$. For every 
$i \in \mathbb{N}$ put $n_{i}=\left\lceil 1 / t_{i}\right\rceil$ and write $I$ as the union of a collection $\mathcal{B}_{i}$ consisting of $n_{i}$ intervals, each with length equal to $t_{i}$. We have

$$
\begin{aligned}
m_{h}(I) & \leq \lim _{i \rightarrow \infty} \sum_{B \in \mathcal{B}_{i}} h(\operatorname{diam}(B))=\lim _{i \rightarrow \infty} n_{i} h\left(t_{i}\right) \\
& \leq \lim _{i \rightarrow \infty}\left(1+t_{i}\right) \frac{h\left(t_{i}\right)}{t_{i}}=0 .
\end{aligned}
$$

Assume now that $\liminf \operatorname{i\searrow 0}_{t} h(t) / t=0$. We define $h^{\prime}(t)=h(t) / t$ for $0<t \leq t_{0}$ and $h^{\prime}(0)=0$ and we apply Corollary 2.8 to $m_{h^{\prime}}$ yielding a nonextendable $A \in \mathcal{K}\left(S^{1}\right)$ with $m_{h^{\prime}}(A)=0$. We will prove that $m_{h}(A \times A)=$ 0 , which violates the premise of the proposition. Let $0<\varepsilon<\min \left\{t_{0}, 1\right\}$ and select a collection $\left\{A_{1}, A_{2}, \ldots\right\}$ of subsets of $S^{1}$ such that $A \subset \bigcup_{i=1}^{\infty} A_{i}$, $\operatorname{diam}\left(A_{i}\right)<\varepsilon$, and $\sum_{i=1}^{\infty} h^{\prime}\left(\operatorname{diam}\left(A_{i}\right)\right) \leq \varepsilon$. Put $a_{i}=\operatorname{diam}\left(A_{i}\right)$ for each $i \in \mathbb{N}$. Since $\liminf \operatorname{in}_{t} h^{\prime}(t)=0$ we may assume that every $a_{i}$ is positive.

Define for every $i$ the natural number $n_{i}=\left\lceil 2 \pi \sqrt{2} / a_{i}\right\rceil$. Partition $S^{1}$ into $n_{i}$ sets $D_{i}^{1}, \ldots, D_{i}^{n_{i}}$ each with diameter no greater than $a_{i} / \sqrt{2}$. Write every $A_{i}$ as the union of two sets $B_{i}$ and $C_{i}$ with diameter at most $a_{i} / \sqrt{2}$. Note that in $\mathbb{R}^{4}, \operatorname{diam}\left(B_{i} \times D_{i}^{j}\right) \leq a_{i}$, and find a $\widetilde{B}_{i}^{j}$ such that $B_{i} \times D_{i}^{j} \subset \widetilde{B}_{i}^{j}$ and $\operatorname{diam}\left(\widetilde{B}_{i}^{j}\right)=a_{i}$. Analogously, let $C_{i} \times D_{i}^{j} \subset \widetilde{C}_{i}^{j}$ and $\operatorname{diam}\left(\widetilde{C}_{i}^{j}\right)=a_{i}$.

Note that

$$
\mathcal{D}=\left\{\widetilde{B}_{i}^{j}: i \in \mathbb{N}, 1 \leq j \leq n_{i}\right\} \cup\left\{\widetilde{C}_{i}^{j}: i \in \mathbb{N}, 1 \leq j \leq n_{i}\right\}
$$

is a countable covering of $A \times A$ with sets of diameter less than $\varepsilon$. Consider the sum

$$
\begin{aligned}
\sum_{D \in \mathcal{D}} h(\operatorname{diam}(D)) & =\sum_{i=1}^{\infty} \sum_{j=1}^{n_{i}}\left(h\left(\operatorname{diam}\left(\widetilde{B}_{i}^{j}\right)\right)+h\left(\operatorname{diam}\left(\widetilde{C}_{i}^{j}\right)\right)\right) \\
& =\sum_{i=1}^{\infty} \sum_{j=1}^{n_{i}} 2 a_{i} h^{\prime}\left(a_{i}\right)=\sum_{i=1}^{\infty} 2 n_{i} a_{i} h^{\prime}\left(a_{i}\right) \\
& \leq \sum_{i=1}^{\infty} 2\left(2 \pi \sqrt{2}+a_{i}\right) h^{\prime}\left(a_{i}\right) \leq 2(2 \pi \sqrt{2}+1) \sum_{i=1}^{\infty} h^{\prime}\left(a_{i}\right) \\
& \leq 2(2 \pi \sqrt{2}+1) \varepsilon .
\end{aligned}
$$

Since $\varepsilon$ can be chosen arbitrarily small, $m_{h}(A \times A)=0$ and the proposition is proved.

The following proposition improves upon [2]. Let $\sigma$ stand for the arc length measure on $S^{1}$.

Proposition 2.10. If $A$ is a measurable and extendable subset of $S^{1}$ then $\sigma(A)=0$. 
Proof. Let $A$ be a subset of $S^{1} \cap B$ where $\sigma(A)>0$ and $B$ is a two point set. According to [7, 7.12] almost every $u \in A$ has the property

$$
\lim _{\varepsilon \searrow 0} \frac{\sigma\left(A \cap U_{\varepsilon}(u)\right)}{\sigma\left(U_{\varepsilon}(u)\right)}=1 .
$$

Let $u$ be such a Lebesgue point and let $\ell$ be the tangent line to $S^{1}$ at $u$. Since $B$ is a two point set we can find a $v \in B \cap \ell$ such that $v \neq u$. The two tangent lines to $S^{1}$ through $v$ divide the circle into two open segments $E$ and $F$ as in Figure 5 .

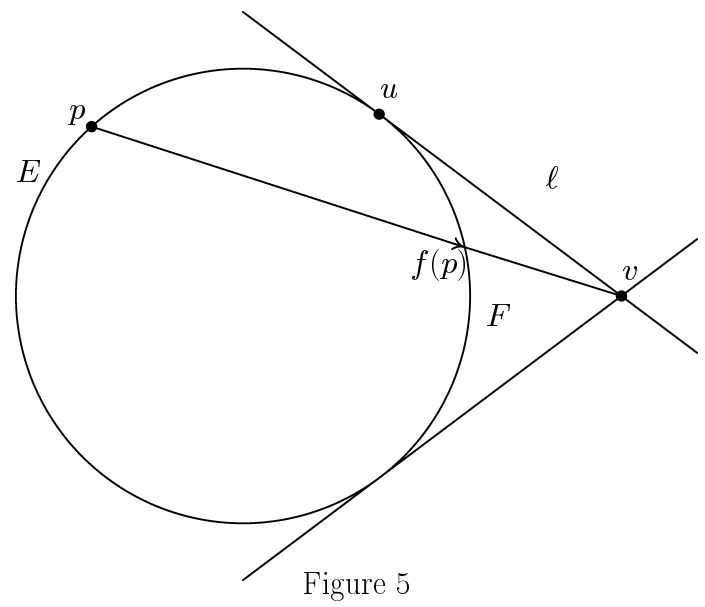

Let $p$ be an element of $E$ and let $f: E \rightarrow F$ be the radial projection centered at $v$. Note that $f$ is a contraction so $\sigma(f(C)) \leq \sigma(C)$ for any measurable $C \subset E$. (Since a line has the same angle of intersection with a circle at both points the contraction factor of $f$ at $p$ is simply the ratio $\frac{|f(p)-v|}{|p-v|}$.) Consequently, we have

$$
\begin{gathered}
\lim _{p \rightarrow u} \frac{\sigma([u, p])}{\sigma([f(p), u])}=\lim _{p \rightarrow u} \frac{\sigma([u, p])}{\int_{u}^{p} \frac{|f(q)-v|}{|q-v|} d \sigma(q)}=\lim _{p \rightarrow u} \frac{|p-v|}{|f(p)-v|}=1, \\
\lim _{p \rightarrow u} \frac{\sigma([f(p), u] \backslash f(A \cap E))}{\sigma([u, p])} \leq \lim _{p \rightarrow u} \frac{\sigma([u, p] \backslash A)}{\sigma([u, p])}=0 .
\end{gathered}
$$

Note that

$$
\begin{aligned}
\lim _{p \rightarrow u} \frac{\sigma([f(p), u] \backslash(A \cap f(A \cap E)))}{\sigma([f(p), u])} \\
\leq \lim _{p \rightarrow u} \frac{\sigma([f(p), u] \backslash A)}{\sigma([f(p), u])} \\
\quad+\lim _{p \rightarrow u} \frac{\sigma([f(p), u] \backslash f(A \cap E))}{\sigma([u, p])} \cdot \lim _{p \rightarrow u} \frac{\sigma([u, p])}{\sigma([f(p), u])}=0 .
\end{aligned}
$$


This result obviously implies that $\sigma(A \cap f(A \cap E))>0$ and we can choose a $p \in A \cap E$ such that $f(p) \in A$. Then $p, f(p)$, and $v$ are three collinear elements of the two point set $B$ and we have arrived at the contradiction that proves the proposition.

The following observation is based on a suggestion by W. Rudin.

Proposition 2.11. If $C$ is a partial two point set with $\operatorname{dim}(C)>0$ then every dense $G_{\delta}$-subset of $C$ is nonextendable.

Proof. Let $C$ be a partial two point set with $\operatorname{dim}(C)>0$. According to Kulesza [3, Theorem 1], $C$ contains an arc, so let $\alpha: I \rightarrow C$ be an imbedding. Kulesza also shows in the proof of [3, Lemma 3] that there is a line $\ell$ that meets $C$ in a single point $u \in \alpha((0,1))$ such that the $\operatorname{arc} \alpha(I)$ is contained in one of the half-planes determined by $\ell$. Let $r \in(0,1)$ be such that $u=\alpha(r)$. Assume that $A$ is a dense $G_{\delta}$-subset of $C$ which is contained in some two point set $B$.

There exists a point $v \in B \cap \ell$ which is distinct from $u$. By connectedness we infer that the line through $v$ and $\alpha(0)$ intersects $\alpha((r, 1])$ or that the line through $v$ and $\alpha(1)$ intersects $\alpha((r, 0])$. If necessary we shorten the arc on one end so that we obtain the collinearity of $\alpha(0), \alpha(1)$, and $v$. By connectedness and the fact that $\alpha(I)$ is a partial two point set we find that for every $p \in \alpha([0, r])$ the line through $p$ and $v$ intersects the arc $\alpha([r, 1])$ in precisely one point, which we denote by $f(p)$. By compactness $f: \alpha([0, r]) \rightarrow \alpha([r, 1])$ is a homeomorphism. Since $C$ is a partial two point set every line through $v$ and an arbitrary point $p \in \alpha((0, r))$ intersects $C$ only in the points $p$ and $f(p)$. Consequently, $E=\alpha((0, r))$ and $F=\alpha((r, 1))$ are open subsets of $C$ and hence $E \cap A$ and $F \cap A$ are dense $G_{\delta}$-subsets of $E$ and $F$ respectively. So we see that $E \cap A \cap f^{-1}(F \cap A)$ is a dense $G_{\delta}$ in $E$ and we may select a $p \in E \cap A$ such that $f(p) \in A$. Then $p, f(p)$, and $v$ are three collinear elements of the two point set $B$, a contradiction.

The following generalization of Proposition 2.10 seems reasonable:

CONJECTURE. Every $\sigma$-compact subset of a two point set has Hausdorff 1-measure zero.

We will find in the next section that the existence of extendable elements of $\mathcal{K}\left(S^{1}\right)$ with positive Hausdorff dimension and capacity is consistent with ZFC.

3. Extendable sets. Several of the results in this section use Martin's Axiom. MA is weaker than the continuum hypothesis and it implies that no compact metric space can be written as the union of less than $\mathfrak{c}=2^{\omega}$ nowhere dense subsets. For more information on MA see e.g. [10]. 
We call a partial two point set $A$ a pre-2-point set if every line $\ell$ in the plane such that $\ell \backslash \mathfrak{L}(A)$ is nowhere dense in $\ell$ intersects $A$ in two points. According to Kulesza [3] every two point set is zero-dimensional. Kulesza shows in effect that if $A$ is a partial two point set with $\operatorname{dim}(A)>0$ then there is a line $\ell \subset \mathfrak{L}(A)$ that intersects $A$ in only one point (see the proof of Lemma 3 in [3]). Note that this result also proves that every pre-2-point set is zero-dimensional.

TheOREM 3.1. (MA) Every $\sigma$-compact pre-2-point set is extendable.

Proof. Let $A$ be a $\sigma$-compact pre-2-point set. Consider the $\sigma$-compactum $E=\{(u, v) \in A \times A: u \neq v\} \times \mathbb{R}$ and the map $g: E \rightarrow \mathbb{R}^{2}$ defined by $g(u, v, t)=u+t(v-u)$. Observe that $\mathfrak{L}(A)=g(E)$ and hence the set is $\sigma$-compact.

Let $\left\{\ell_{\alpha}: \alpha<\mathfrak{c}\right\}$ enumerate the lines in the plane. We shall construct by transfinite induction a nondecreasing sequence $\left(C_{\alpha}\right)_{\alpha \leq \mathfrak{c}}$ of subsets of $\mathbb{R}^{2} \backslash A$ with induction hypotheses:

(1) $\left|C_{\alpha}\right| \leq|\alpha|+\omega$

(2) $A \cup C_{\alpha}$ is a partial two point set.

Put $C_{0}=\emptyset$ and if $\lambda \leq \mathfrak{c}$ is a limit ordinal then $C_{\lambda}=\bigcup_{\alpha<\lambda} C_{\alpha}$. Let $\alpha$ be a fixed ordinal $<\mathfrak{c}$ and consider $C_{\alpha}$ and $\ell_{\alpha}$. Assume for the moment that $\left(A \cup C_{\alpha}\right) \cap \ell_{\alpha}$ contains at most one point. Let $I$ be a nondegenerate compact interval in $\ell_{\alpha}$ such that $I \subset \operatorname{cl}\left(\ell_{\alpha} \backslash \mathfrak{L}(A)\right)$. Note that since $I \cap \mathfrak{L}(A)$ is $\sigma$-compact it is a category I set in $I$.

Let $u$ be a point in $C_{\alpha}$. If $u \in \ell_{\alpha}$ then we put $B_{u}=\{u\} \cap I$; otherwise we define

$$
B_{u}=\{v \in I: L(u, v) \cap A \neq \emptyset\} .
$$

Let $u \notin \ell_{\alpha}$ and note that $D=\bigcup\{L(u, v): v \in I\}$ is closed. Since $A$ is a zero-dimensional $\sigma$-compactum, $D \cap A$ can be written as a countable union $\bigcup_{i=1}^{\infty} D_{i}$ of zero-dimensional compacta. Let $f: D \backslash\{u\} \rightarrow I$ be the projection defined by $\{f(z)\}=L(u, z) \cap I$. Since $D_{i} \cup\{u\}$ is a subset of $A \cup C_{\alpha}$ it is also a partial two point set and $f \mid D_{i}$ is one-to-one and hence an imbedding. Consequently, $B_{u}=\bigcup_{i=1}^{\infty} f\left(D_{i}\right)$ is a countable union of zero-dimensional compacta. So $B_{u}$ is of category I in $I$ for each $u \in C_{\alpha}$.

Consider now two distinct points $u$ and $v$ in $C_{\alpha}$. Since $u$ and $v$ do not both lie on $\ell_{\alpha}$ the set $B_{u v}=L(u, v) \cap I$ contains at most one point. Note that

$$
\mathcal{D}=\{I \cap \mathfrak{L}(A)\} \cup\left\{B_{u}: u \in C_{\alpha}\right\} \cup\left\{B_{u v}: u, v \in C_{\alpha} \text { and } u \neq v\right\}
$$

is a collection of category I subsets of $I$ with $|\mathcal{D}| \leq|\alpha|+\omega<\mathfrak{c}$ and that $\bigcup \mathcal{D}=I \cap \mathfrak{L}\left(A \cup C_{\alpha}\right)$. Martin's Axiom implies that $I \backslash \bigcup \mathcal{D}$ is dense in $I$ and we can select two distinct points $a$ and $b$ in that dense set. 
Since $A \cup C_{\alpha}$ is a partial two point set we may define

$$
C_{\alpha+1}= \begin{cases}C_{\alpha} \cup\{a, b\} & \text { if }\left|\left(A \cup C_{\alpha}\right) \cap \ell_{\alpha}\right|=0, \\ C_{\alpha} \cup\{a\} & \text { if }\left|\left(A \cup C_{\alpha}\right) \cap \ell_{\alpha}\right|=1, \\ C_{\alpha} & \text { if }\left|\left(A \cup C_{\alpha}\right) \cap \ell_{\alpha}\right|=2 .\end{cases}
$$

By the construction it is obvious that $\left|C_{\alpha+1}\right| \leq|\alpha+1|+\omega$ and that $A \cup C_{\alpha+1}$ is a partial two point set which intersects $\ell_{\alpha}$ in two points. Then $A \cup C_{\mathfrak{c}}$ is a two point set.

THEOREM 3.2. (MA) Any $\sigma$-compact partial two point set $A$ with $\mathcal{H}^{1}(\mathfrak{B}(A))=0$ is extendable.

Proof. First we show that $A$ is zero-dimensional. Assume that $\operatorname{dim}(A)$ $\geq 1$. Select a $u \in A$ such that $u \neq 0$. Then $A \backslash\{u\}$ is a $\sigma$-compactum with $\operatorname{dim} \geq 1$ and hence it contains a compactum $B$ with $\operatorname{dim}(B) \geq 1$. Select a nontrivial continuum $C \subset B$. Define the continuous map $h: C \rightarrow$ $\mathbb{R}^{2}$ by $h(v)=p(\varphi(v-u), v)$. So $h(C)$ is a continuum in $\mathfrak{B}(A)$ and hence $\operatorname{diam}(h(C)) \leq \mathcal{H}^{1}(h(C)) \leq \mathcal{H}^{1}(\mathfrak{B}(A))=0$. Let $v$ and $w$ be distinct elements of $C$ and note that $h(v)=h(w) \in L(u, v) \cap L(u, w)$. Since $A$ is a partial two point set, $u, v$, and $w$ are not collinear, which implies that $L(u, v) \cap L(u, w)=$ $\{u\}$. So $u=h(v)=h(w)$, which means since $u \neq 0$ that $v$ and $w$ lie on the line through $u$ that is perpendicular to the line through 0 and $u$. So $u, v$, and $w$ are collinear, a contradiction.

In order to show that $A$ is a pre-2-point set let $\ell$ be a line that intersects $A$ in less than two points. We shall prove that $\mathfrak{L}(A) \cap \ell$ is of category I in $\ell$. By rotating the $x y$-axes we can arrange that $\ell$ is the line $x=a$. Define a function $f$ from $D=\left\{(x, y) \in \mathbb{R}^{2}: y \neq 0\right\}$ to $\ell$ by $f(x, y)=\left(a,\left(x^{2}+y^{2}-a x\right) / y\right)$. Note that if $u$ is a vector from $D$ then $u$ and $f(u)-u$ are perpendicular. Since $A$ is $\sigma$-compact, so are $\mathfrak{B}(A)$ and $\mathfrak{B}(A) \cap D$. Write $P=\mathfrak{B}(A) \cap D=\bigcup_{i=1}^{\infty} P_{i}$ where the $P_{i}$ 's are compact. Since $f$ is analytic the maps $f \mid P_{i}$ are Lipschitz and we have $\mathcal{H}^{1}\left(f\left(P_{i}\right)\right)=0$ because $\mathcal{H}^{1}\left(P_{i}\right) \leq \mathcal{H}^{1}(\mathfrak{B}(A))=0$. Consequently, $f(P)$ is a countable union of zero-dimensional compacta and hence it is a category I set in $\ell$.

Consider the case $a=0$, i.e. $\ell$ is the $y$-axis. Let $u \neq 0$ be an element of $\mathfrak{L}(A) \cap \ell$. There are $v$ and $w$ in $A$ such that $u \in L(v, w)$. Since $\ell$ intersects $A$ in at most one point we know that $L(u, v)$ is not vertical. Note that $z=p(\varphi(v-w), v)$ is an element of $P$ such that $f(z)=u$. We may conclude that $\mathfrak{L}(A) \cap \ell$ is a subset of $\{0\} \cup f(P)$ and hence it is a category I set in $\ell$.

Assume now that $a \neq 0$. Consider the zero-dimensional $\sigma$-compactum $A^{\prime}=A \cap(D \cup\{0\})$. Define the set

$$
R=\left\{(u, v) \in A^{\prime} \times A^{\prime}: u, v, 0 \text { collinear and } u \neq v\right\} .
$$

Since $R$ is the intersection of an open and a closed subset of $A^{\prime} \times A^{\prime}$ it is also zero-dimensional and $\sigma$-compact. Since $R$ is disjoint from the diagonal 
of $A^{\prime} \times A^{\prime}$ we can write $R$ as a countable union of compacta $R_{1}, R_{2}, \ldots$, where $(u, v) \in R_{i}$ implies $(v, u) \notin R_{i}$. Define the continuous map $g: R \rightarrow \ell$ by $\{g(u, v)\}=L(u, v) \cap \ell$. Let $(u, v)$ and $(w, z)$ be elements of $R_{i}$ such that $g(u, v)=g(w, z)$. Then $L(u, v)$ and $L(w, z)$ have the origin and $g(u, v)$ in common, so they coincide. Since $A$ is a partial two point set we have $\{u, v\}=\{w, z\}$ and hence $(u, v)=(w, z)$. Consequently, $g \mid R_{i}$ is one-to-one and an imbedding. This implies that $g(R)$ is a countable union of zerodimensional compacta and hence a category I set in $\ell$. Let $v$ and $w$ be elements of $A$ such that $L(v, w)$ contains some $u \in \ell$. If $L(v, w)$ does not go through the origin then by the same argument as given above we have $u \in f(P)$. If $L(v, w)$ does contain the origin then $(v, w) \in R$ and $g(v, w)=u$. So we may conclude that $\mathfrak{L}(A) \cap \ell \subset f(P) \cup g(R)$ and hence it is a category I set in $\ell$.

According to Section 2 there exist nonextendable compact partial two point sets with Hausdorff 1-measure zero (or even with Hausdorff dimension zero). On the other hand, we have:

Theorem 3.3. (MA) Any $\sigma$-compact partial two point set $A$ with $\mathcal{H}^{1}(A \times A)=0$ is extendable.

Proof. We assume that $A \times A$ is equipped with the standard metric it inherits from $\mathbb{R}^{4}$. Let $\Delta=\left\{(u, v) \in \mathbb{R}^{2} \times \mathbb{R}^{2}: u=v\right\}$ and define the $\operatorname{map} f: \mathbb{R}^{4} \backslash \Delta \rightarrow \mathbb{R}^{2}$ by $f(u, v)=p(\varphi(u-v), u)$ for $u, v \in \mathbb{R}^{2}, u \neq v$. Obviously, $B=A \times A \backslash \Delta$ can be written as a countable union of compacta $B_{1}, B_{2}, \ldots$ Since $f$ is analytic $f \mid B_{i}$ is Lipschitz and we may conclude that $\mathcal{H}^{1}\left(f\left(B_{i}\right)\right)=0$ because $\mathcal{H}^{1}\left(B_{i}\right) \leq \mathcal{H}^{1}(A \times A)=0$ for each $i \in \mathbb{N}$. By $\sigma$-additivity of Hausdorff measures we have $\mathcal{H}^{1}(\mathfrak{B}(A))=\mathcal{H}^{1}\left(\bigcup_{i=1}^{\infty} f\left(B_{i}\right)\right)$ $=0$ and Theorem 3.2 applies.

According to Corollary 2.7 there exist nonextendable elements $A$ of $\mathcal{K}\left(S^{1}\right)$ such that $\operatorname{dim}_{\mathrm{H}}(A \times A) \leq 1$. So Theorem 3.3 is sharp in the sense that we cannot replace the condition $\mathcal{H}^{1}(A \times A)=0$ by $\mathcal{H}^{s}(A \times A)=0$ for all $s>1$. In fact, the following result, which combines Theorem 3.3 and Proposition 2.9 , shows that $\mathcal{H}^{1}$ is the optimum choice among the $h$-measures.

THEOREM 3.4. (MA) If $h$ is an arbitrary function from $\left[0, t_{0}\right]$ into $[0, \infty)$ then the following statements are equivalent:

(1) every $\sigma$-compact partial two point set $A$ with $m_{h}(A \times A)=0$ is extendable,

(2) $m_{h}(X)=0$ implies $\mathcal{H}^{1}(X)=0$ for every $X$,

(3) every $A \in \mathcal{K}\left(S^{1}\right)$ with $m_{h}(A \times A)=0$ is extendable,

(4) $\liminf \operatorname{in}_{t} h(t) / t>0$. 
Note that $\mathcal{H}^{1}(A \times A)$ is an upper semicontinuous function of $A$ that vanishes on finite sets. Consequently, the null-subadditivity condition in Theorem 2.3 is essential and we have

Corollary 3.5. (MA) If $X$ is any partial two point set then there exists a dense $G_{\delta}$-subset of $\mathcal{K}(X)$ consisting entirely of extendable sets.

So the nonextendable sets in $\mathcal{K}(X)$ form merely a category I set.

We now describe a standard procedure for constructing a Cantor set $C$. Let $C_{0}$ be a continuum. As step 1 we replace $C_{0}$ by $n_{1}$ disjoint subcontinua $C_{1}^{1}, \ldots, C_{1}^{n_{1}}$. In general, for $i \in \mathbb{N}$ we replace every $C_{i-1}^{j}$, for $1 \leq j \leq$ $n_{1} \ldots n_{i-1}$, by $n_{i}$ disjoint subcontinua. This produces a set $C_{i}=C_{i}^{1} \cup \ldots \cup C_{i}^{k}$, where $k=n_{1} \ldots n_{i}$. Define

$$
d_{i}=\max \left\{\operatorname{diam}\left(C_{i}^{j}\right): 1 \leq j \leq n_{1} \ldots n_{i}\right\}
$$

and $r_{i}=d_{i} / d_{i-1}$. Then $C$ is the intersection $\bigcap_{i=0}^{\infty} C_{i}$.

Corollary 3.6. (MA) Let $C$ be a planar Cantor set as constructed above. If $C$ is a partial two point set such that $\prod_{i=1}^{\infty} n_{i}^{2} r_{i}=0$ then $C$ is extendable.

Pr o o f. Consider the covering

$$
\mathcal{B}_{i}=\left\{C_{i}^{j} \times C_{i}^{k}: 1 \leq j, k \leq n_{1} \ldots n_{i}\right\}
$$

of $C \times C$. Let $d_{0}=\operatorname{diam}\left(C_{0}\right)$ and let $\operatorname{mesh}\left(\mathcal{B}_{i}\right)=\max \left\{\operatorname{diam}\left(B_{i}\right): B \in \mathcal{B}_{i}\right\}$. We have

$$
\lim _{i \rightarrow \infty} \operatorname{mesh}\left(\mathcal{B}_{i}\right) \leq \lim _{i \rightarrow \infty} \sqrt{2} d_{0} r_{1} \ldots r_{i} \leq \sqrt{2} d_{0} \prod_{i=1}^{\infty} n_{i}^{2} r_{i}=0 .
$$

In addition,

$$
\begin{aligned}
\lim _{i \rightarrow \infty} \sum_{B \in \mathcal{B}_{i}} \operatorname{diam}(B) & \leq \lim _{i \rightarrow \infty}\left|\mathcal{B}_{i}\right| \operatorname{mesh}\left(\mathcal{B}_{i}\right) \\
& \leq \lim _{i \rightarrow \infty}\left(n_{1} \ldots n_{i}\right)^{2} \sqrt{2} d_{0} r_{i} \ldots r_{i}=\sqrt{2} d_{0} \prod_{i=1}^{\infty} n_{i}^{2} r_{i}=0 .
\end{aligned}
$$

Consequently, $\mathcal{H}^{1}(C \times C)=0$ and we may apply Theorem 3.3.

For instance, if we start with an interval in $S^{1}$ and we obtain $C_{i+1}$ from $C_{i}$ by deleting the middle three fifths of every interval of $C_{i}$ then $n_{i}=2$ and $r_{i}=1 / 5$. Consequently, $\prod_{i=1}^{\infty} n_{i}^{2} r_{i}=(4 / 5)^{\infty}=0$ and the resulting Cantor set is extendable to a two point set. In addition, one may verify that the set has positive Hausdorff dimension and hence positive logarithmic capacity.

Corollary 3.6 gives us many Cantor sets that are extendable to two point sets provided that Martin's Axiom is valid, suggesting the following question: is the existence of a two point set that contains a Cantor set provable in 
ZFC? Noting that every dense $G_{\delta}$ in $\mathcal{K}\left(S^{1}\right)$ contains Cantor sets we have an affirmative answer in the following result.

Proposition 3.7. There exists a dense $G_{\delta}$-subset of $\mathcal{K}\left(S^{1}\right)$ consisting entirely of extendable sets.

We need some definitions (cf. [5, §VIII.1]) and two technical lemmas. If $A$ is a subset of a field then the subfield generated by $A$ is denoted by $\mathbb{Q}(A)$. A subset $A$ of a field is called algebraically independent if every $x \in A$ is transcendental over the field $\mathbb{Q}(A \backslash\{x\})$. A transcendence base $A$ for a field $F$ is an algebraically independent subset such that $F$ is algebraic in $\mathbb{Q}(A)$. The transcendence degree of a field $F$ over a subset $A$ equals $\min \{|B|: B \subset$ $F$ and $F$ is algebraic in $\mathbb{Q}(A \cup B)\}$.

Lemma 3.8. If $X$ and $Y$ are spaces, $F$ is a closed subset of $X, O$ is an open subset of $Y$, and $f: F \rightarrow Y$ is continuous then the set $\mathcal{O}=\{K \in$ $\mathcal{K}(X): f(K \cap F) \subset O\}$ is open in $\mathcal{K}(X)$.

Proof. The set $f^{-1}(O)$ is open in $F$ and hence $f^{-1}(O) \cup(X \backslash F)$ is open in $X$. Note that $\mathcal{O}=\left\{K \in \mathcal{K}(X): K \subset f^{-1}(O) \cup(X \backslash F)\right\}$ and hence $\mathcal{O}$ is obviously open.

Lemma 3.9. If $D$ is an algebraically independent compact subset of $\mathbb{R}$ such that $\mathbb{R}$ has infinite transcendence degree over $D$ then

$$
\mathcal{G}_{D}=\{C \in \mathcal{K}(\mathbb{R} \backslash D): D \cup C \text { is algebraically independent }\}
$$

is a dense $G_{\delta}$-subset of $\mathcal{K}(\mathbb{R})$.

Proof. Let $D$ be such a subset of $\mathbb{R}$ and let $p_{1 n}, p_{2 n}, \ldots$ be an enumeration of all nonzero polynomials in $n$ variables with integral coefficients. Let $\Delta_{n}$ be the closed subset of $\mathbb{R}^{n}$ that consists of all points with at least two identical coordinates. Define for $m, n \in \mathbb{N}$ the following set of compacta:

$$
G_{m n}=\left\{C \in \mathcal{K}(\mathbb{R} \backslash D): 0 \notin p_{m n}\left((D \cup C)^{n} \backslash \Delta_{n}\right)\right\} .
$$

Note that $\mathcal{G}_{D}=\bigcap\left\{G_{m n}: m, n \in \mathbb{N}\right\}$. Write $\mathbb{R}^{n} \backslash \Delta_{n}$ as a countable union of compacta $K_{1} \subset K_{2} \subset \ldots$ Fix $m, n \in \mathbb{N}$. Consider for each $i \in \mathbb{N}$ the set

$$
U_{i}=\left\{C \in \mathcal{K}(\mathbb{R} \backslash D): 0 \notin p_{m n}\left((D \cup C)^{n} \cap K_{i}\right)\right\} .
$$

According to Lemma 3.8 every $U_{i}$ is open in $\mathcal{K}(\mathbb{R})$. Since $G_{m n}=\bigcap_{i=1}^{\infty} U_{i}$ it is a $G_{\delta}$-set and hence $\mathcal{G}_{D}$ is a $G_{\delta^{-}}$-set as well.

Let $F=\left\{x_{1}, \ldots, x_{n}\right\}$ be a finite set in $\mathbb{R}$ and let $\varepsilon>0$. We define by induction sets $C_{0}, \ldots, C_{n}$ with $\left|C_{i}\right|=i$. Put $C_{0}=\emptyset$ and let $0 \leq i<n$. Since $\mathbb{R}$ has infinite transcendence degree over $D$ there exists an $a \in \mathbb{R}$ that is transcendental over $\mathbb{Q}\left(D \cup C_{i}\right)$. Consequently, every nonzero element of the dense set $\mathbb{Q} a$ is transcendental over $\mathbb{Q}\left(D \cup C_{i}\right)$ and we can select a $y_{i+1} \in \mathbb{Q} a \backslash\{0\}$ that is $\varepsilon$-close to $x_{i+1}$. Put $C_{i+1}=C_{i} \cup\left\{y_{i+1}\right\}$. Note that 
$C_{n}$ is $\varepsilon$-close to $F$ in the Hausdorff metric and that $C_{n} \in \mathcal{G}_{D}$. So the closure of $\mathcal{G}_{D}$ contains all finite sets and hence it is $\mathcal{K}(\mathbb{R})$.

Proof of Proposition 3.7. Let $A$ be an algebraically independent Cantor set in $\mathbb{R}$. The existence of such sets is known and follows if we substitute $D=\emptyset$ in Lemma 3.9. Let $D$ be a Cantor set that is a proper subset of $A$. Then the transcendence degree of $\mathbb{R}$ over $\mathbb{Q}(D)$ is $\mathfrak{c}=|A \backslash D|$; see $[5$, Theorem VIII.1.1]. By Lemma 3.9, $\mathcal{G}_{D}$ is a dense $G_{\delta}$ in $\mathcal{K}(\mathbb{R})$. Define $\pi_{1}, \pi_{2}: \mathbb{R}^{2} \rightarrow \mathbb{R}$ by $\pi_{1}(x, y)=x$ and $\pi_{2}(x, y)=y$. It is easily seen that $G=\left\{C \in \mathcal{K}(S)^{1}\right.$ : $\left.\pi_{1}(C) \in \mathcal{G}_{D}\right\}$ is a dense $G_{\delta}$ in $\mathcal{K}\left(S^{1}\right)$.

We show that every $C \in G$ is extendable to a two point set. $\pi_{1}(C) \cup D$ is algebraically independent, so we can extend this set to a transcendence base $B$ for $\mathbb{R}$. Let $\left\{\ell_{\alpha}: \alpha<\mathfrak{c}\right\}$ enumerate the lines in the plane. We shall construct by transfinite induction a nondecreasing sequence $\left(E_{\alpha}\right)_{\alpha \leq \mathfrak{c}}$ of subsets of $\mathbb{R}^{2} \backslash C$ with the following induction hypotheses:

(1) $\left|E_{\alpha}\right| \leq|\alpha|+\omega$

(2) $C \cup E_{\alpha}$ is a partial two point set.

Put $E_{0}=\emptyset$ and if $\lambda \leq \mathfrak{c}$ is a limit ordinal then $E_{\lambda}=\bigcup_{\alpha<\lambda} E_{\alpha}$. Let $\alpha$ be a fixed ordinal $<\mathfrak{c}$ and consider $E_{\alpha}$ and $\ell_{\alpha}$. If $\left(C \cup E_{\alpha}\right) \cap \ell_{\alpha}$ contains two points then $E_{\alpha+1}=E_{\alpha}$. Assume now that $\left|\left(C \cup E_{\alpha}\right) \cap \ell_{\alpha}\right| \leq 1$ and that $\ell_{\alpha}$ is the graph of $a x+b y=c$. Consider the set $Z=\mathfrak{L}\left(C \cup E_{\alpha}\right) \cap \ell_{\alpha}$. If $(x, y) \in Z$ then there exist two distinct points $\left(x_{1}, y_{1}\right),\left(x_{2}, y_{2}\right) \in C \cup E_{\alpha}$ such that $(x, y)$ is the point of intersection of $\ell_{\alpha}$ and the line through $\left(x_{1}, y_{1}\right)$ and $\left(x_{2}, y_{2}\right)$. Consequently, $x$ and $y$ are elements of the field $\mathbb{Q}\left(\left\{x_{1}, x_{2}, y_{1}, y_{2}, a, b, c\right\}\right)$. Since $\left|E_{\alpha}\right| \leq|\alpha|+\omega$ we can find a $B^{\prime} \subset B$ such that $\left|B^{\prime}\right| \leq|\alpha|+\omega$ and $\pi_{1}\left(E_{\alpha}\right) \cup \pi_{2}\left(E_{\alpha}\right) \cup\{a, b, c\}$ is algebraic in $\mathbb{Q}\left(B^{\prime}\right)$. Since $S^{1}$ is an algebraic curve, $\pi_{2}(C)$ is algebraic in $\mathbb{Q}\left(\pi_{1}(C)\right)$. As the set of points that are algebraic in a given field is itself a field we may conclude that $\pi_{1}(Z) \cup \pi_{2}(Z)$ is algebraic in $\mathbb{Q}\left(\pi_{1}(C) \cup B^{\prime}\right)$. Since $\left|B^{\prime}\right|<\mathfrak{c}$ we can select two distinct points $u$ and $v$ in $\ell_{\alpha}$ such that at least one of their coordinates is in $D \backslash B^{\prime}$ and hence transcendental over $\mathbb{Q}\left(\pi_{1}(C) \cup B^{\prime}\right)$. Consequently, $u$ and $v$ are not in $\mathfrak{L}(C \cup$ $\left.E_{\alpha}\right)$. Putting $E_{\alpha+1}$ equal to $E_{\alpha} \cup\{u\}$ or $E_{\alpha} \cup\{u, v\}$ completes the induction. It is obvious that $C \cup E_{\mathfrak{c}}$ is a two point set.

Note that in this proof we may replace $S^{1}$ by any algebraic curve that is a partial two point set. In fact, it is shown in [1] that Corollary 3.5 is provable in ZFC.

In connection with Proposition 3.7, A. W. Miller asked the following

Question. What is the Borel type of the set of extendable elements of $\mathcal{K}\left(S^{1}\right)$ ?

If we combine Proposition 3.7 with Theorem 2.2 we find 
Proposition 3.10. There exist two extendable elements of $\mathcal{K}\left(S^{1}\right)$ whose union is not extendable to a two point set.

\section{References}

[1] J. J. Dijkstra, Generic partial two-point sets are extendable, Canad. Math. Bull., to appear.

[2] J. J. Dijkstra and J. van Mill, Two point set extensions-a counterexample, Proc. Amer. Math. Soc. 125 (1997), 2501-2502.

[3] J. Kulesza, A two-point set must be zero-dimensional, ibid. 116 (1992), 551-553.

[4] N. S. Landkof, Foundations of Modern Potential Theory, Grundlehren Math. Wiss. 180, Springer, Berlin, 1972.

[5] S. Lang, Algebra, 3rd ed., Addison-Wesley, Reading, 1993.

[6] R. D. Mauldin, Problems in topology arising from analysis, in: Open Problems in Topology, J. van Mill and G. M. Reed (eds.), North-Holland, Amsterdam, 1990, 617-629.

[7] - - On sets which meet each line in exactly two points, Bull. London Math. Soc., to appear.

[8] S. Mazurkiewicz, O pewnej mnogości płaskiej, która ma z każda prosta dwa i tylko dwa punkty wspólne, C. R. Varsovie 7 (1914), 382-384 (in Polish); French transl.: Sur un ensemble plan qui a avec chaque droite deux et seulement deux points communs, in: Stefan Mazurkiewicz, Traveaux de Topologie et ses Applications, PWN, Warszawa, 1969, 46-47.

[9] J. van Mill and G. M. Reed, Open problems in topology, Topology Appl. 62 (1995), 93-99.

[10] M. E. Rudin, Martin's Axiom, in:Handbook of Mathematical Logic, North-Holland, Amsterdam, 1977, 491-501.

[11] W. Rudin, Real and Complex Analysis, 3rd ed., McGraw-Hill, New York, 1987.

[12] M. Tsuji, Potential Theory in Modern Function Theory, Maruzen, Tokyo, 1959.

Department of Mathematics

The University of Alabama

Box 870350

Tuscaloosa, Alabama 35487-0350

U.S.A.

E-mail: jdijkstr@ua1vm.ua.edu

Department of Mathematics

University of Wisconsin

Madison, Wisconsin 53706

U.S.A.

E-mail: tt kunen@math.wisc.edu
Faculteit Wiskunde en Informatica Vrije Universiteit De Boelelaan 1081a 1081 HV Amsterdam, The Netherlands E-mail: vanmill@cs.vu.nl

Received 17 June 1997;

in revised form 28 January 1998 